\title{
The Pivot Energy of Solar Energetic Particles Affecting the Martian Surface Radiation Environment
}

\author{
Jingnan Guo ${ }^{1,2,3}$ (10), Robert F. Wimmer-Schweingruber ${ }^{3}$ (D), Yuming Wang ${ }^{1,2}(\mathbb{D})$, Manuel Grande ${ }^{4}$ (1D), Daniel Matthiä ${ }^{5}$, \\ Cary Zeitlin ${ }^{6}$ (i), Bent Ehresmann ${ }^{7}$ (i), and Donald M. Hassler ${ }^{7}$ (i) \\ ${ }^{1}$ School of Earth and Space Sciences, University of Science and Technology of China, Hefei, People's Republic of China; jnguo@ustc.edu.cn \\ ${ }^{2}$ CAS Center for Excellence in Comparative Planetology, Hefei, People's Republic of China \\ ${ }^{3}$ Institute of Experimental and Applied Physics, Christian-Albrechts-University, Kiel, Germany \\ ${ }^{4}$ University of Aberystwyth, Aberystwyth, UK \\ ${ }^{5}$ German Aerospace Agency, Cologne, Germany \\ ${ }^{7}$ Planetary Science Division, Southwest Research Institute, Boulder, CO, USA \\ Received 2019 August 2; revised 2019 August 23; accepted 2019 August 27; published 2019 September 19
}

\begin{abstract}
Space radiation is a major risk for humans, especially on long-duration missions to outer space, e.g., a manned mission to Mars. Galactic cosmic rays contribute a predictable radiation background; the main risk is due to the highly variable and currently unpredictable flux of solar energetic particles (SEPs). Such sporadic SEP events may induce acute health effects and are thus considered a critical mission risk for future human exploration of Mars. Therefore, it is of the utmost importance to study, model, and predict the surface radiation environment during such events. It is well known that the deep-space SEP differential energy spectrum at high energies is often given by a power law. We use a measurement-validated particle transport code to show that, for large SEP events with proton energy extending above $\sim 500 \mathrm{MeV}$ with a power-law distribution, it is sufficient to measure the SEP flux at a pivot energy of $\sim 300 \mathrm{MeV}$ above the Martian atmosphere to predict the dose rate on the Martian surface. In conjunction with a validation by in situ measurements from the Martian surface, this remarkable simplification and elegant quantification could enable instant predictions of the radiation environment on the surface of Mars upon the onset of large SEP events.
\end{abstract}

Unified Astronomy Thesaurus concepts: Solar energetic particles (1491); Space weather (2037); Cosmic rays (329); Mars (1007); Solar-planetary interactions (1472); Particle physics (2088)

\section{Introduction}

Galactic cosmic rays (GCRs) and solar energetic particles (SEPs) are the two major types of energetic particles in the heliosphere that may impose radiation risks to humans on space and planetary missions. On the surface of Earth, we are mostly protected against such high-energy particles from space thanks to Earth's magnetosphere and our sufficiently thick atmosphere that can deflect and stop the large majority of the high-energy charged particles. However, the surface of Mars is much more exposed to highly energetic particles than Earth because of the lack of a global magnetic field and its very thin atmosphere. With significantly less shielding, exposure to the radiation environment on the surface of Mars remains a major concern and health risk for future human explorers (e.g., Cucinotta \& Durante 2006; Hassler et al. 2014).

GCRs are mainly composed of protons and helium ions (Simpson 1983) and are omnipresent as they arrive in the solar system from interstellar space. They are modulated by heliospheric magnetic fields that evolve dynamically as solar activity varies in time and space, with a well-known $11 \mathrm{yr}$ cycle (e.g., Parker 1958). In contrast to GCRs, SEPs-mainly protons and electrons-are emitted from the Sun and accelerated by sporadic solar eruptions such as flares and/or coronal mass ejection (CME) associated shocks (e.g., Lario 2005). SEP events are often impulsive and could enhance the radiation level significantly, especially at places that are magnetically connected to the particle injection site and where sufficient shielding against radiation is unavailable, such as on the Martian surface (e.g., Guo et al. 2018b). As for near-future human exploration missions to Mars, the immediate forecast (or nowcast) of the radiation environment on the surface of Mars during periods of enhanced solar activity is essential and critical.

Hitherto, SEP measurements on the surface Mars are very scarce and time limited. The Mars Science Laboratory (MSL) rover Curiosity landed on Mars on 2012 August 6 (Grotzinger et al. 2012). Since then, its Radiation Assessment Detector (RAD; Hassler et al. 2012) has been measuring the Mars surface radiation environment that so far has been dominated by the GCR component. To date only six SEP events have been detected on Mars, and of these the 2017 September 10 solar eruption associated SEP event is the most intense event seen by RAD (Ehresmann et al. 2018; Guo et al. 2018a; Hassler et al. 2018; Zeitlin et al. 2018) and is also the first and only solar particle event that caused ground-level enhancement detected at two different planets, Earth and Mars (Guo et al. 2018a). Nevertheless, this event is not intense enough to pose any radiation risk to the health of an astronaut at the ground level of Mars (Zeitlin et al. 2018).

This sparsity of surface data for SEP-induced radiation requires us to resort to sophisticated physics-based modeling thereof. In some previous studies (Matthiä et al. 2016, 2017; Guo et al. 2019a) and as also described in Appendix A, we developed a GEANT4 particle transport model implemented with Martian atmospheric and regolith properties and benchmarked this model using RAD measurements. Here we use this validated model to calculate the surface radiation for different input spectra at the top of the atmosphere. In particular, we 

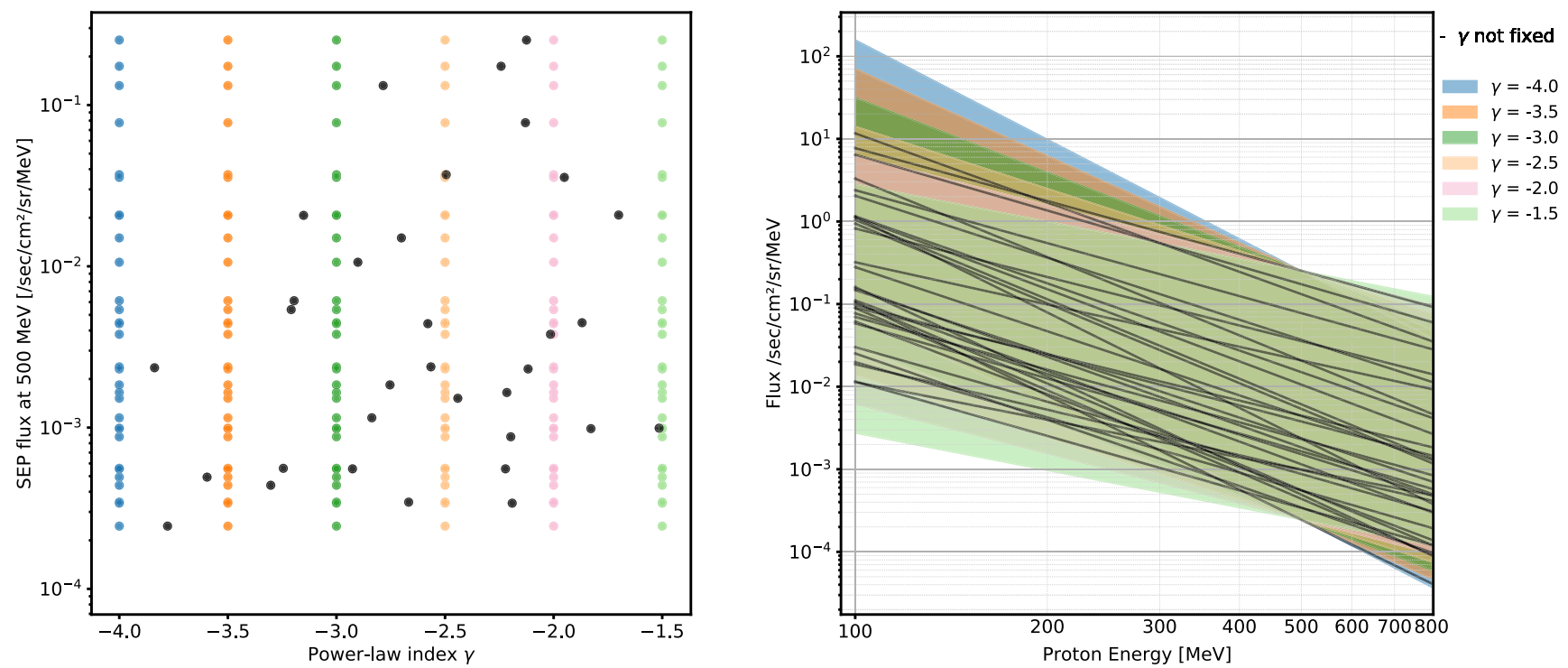

Figure 1. Left: SEP spectral intensity at $500 \mathrm{MeV}$ versus spectral index $\gamma$ of SEP events used in this study. Right: SEP spectra in the range of 100-800 MeV. Black points (left panel) or black lines (right panel) are the power-law spectra of the original 33 events from Kühl et al. (2017). Different colored ranges are the regenerated spectra with each color representing a set of SEP spectra with the same power-law index $\gamma$, but different intensities.

calculate the induced Martian surface radiation by a variety of SEP events with different properties such as their energy range, intensity, power-law index, etc. For the first time, we find a pivot energy $(\sim 300 \mathrm{MeV})$ at which the SEP flux alone can be used to determine the Martian surface dose rate. In other words, with a fixed flux at this pivot energy, the variation of the power-law spectral index does not affect the surface radiation. This finding advances our understanding of the radiation risks during possibly adverse space weather conditions. Together with SEP injection and interplanetary transport models, we can provide instantaneous and quantitative alerts for future human missions at Mars upon the onset of large SEP events at the Sun.

\section{Method}

In this study, we calculate the induced Martian surface radiation by a variety of SEP events with different properties such as their energy range, intensity, power-law index, etc. Then we analyze and correlate the parameterized properties of the SEP spectra and the resulting surface dose rate of each event in order to find a simplified quantification of the Martian surface radiation based on SEP spectral properties. Starting from a list of more than 30 significant SEP events detected in situ at Earth by the Solar and Heliospheric Observatory $(\mathrm{SOHO})$ in $20 \mathrm{yr}$ (Kühl et al. 2017), each event spectrum from 100 to $800 \mathrm{MeV}$ was derived in a $2 \mathrm{hr}$ interval starting 30 minutes after the event onset (in an energy channel covering $100 \mathrm{MeV}$ to $1 \mathrm{GeV}$ ) and then fitted with a power-law distribution of $I(E)=I_{0} E^{\gamma}$, shown as black lines in Figure 1 . The fitted power-law parameters of each event and the goodness of the fit $\left(R^{2}\right.$ mostly larger than 0.9$)$ are listed in Table 3 of Kühl et al. (2017). It is not certain that these events reached Mars, and even if they did, the SEP spectra were probably different from those measured at Earth. This is because the observed SEP spectra and intensity depend on the magnetic connections of the planets/spacecraft to the acceleration/injection locations and also the propagation of SEP particles in the interplanetary space. However, for the purpose of a statistical and parametric study for how different SEP properties may affect the Martian radiation environment, we use these near-Earth spectra at Mars to obtain a better understanding of SEP-induced radiation on Mars.

Based on each fitted SEP spectrum, we calculate its induced secondary spectra and accumulated radiation dose rate on the surface of Mars in a $0.5 \mathrm{~mm}$ water slab (an approximation of thin-skin structures of a human body). In order to quantify the dependence of the surface radiation on SEP properties, we also use SEP spectra with the same spectral power-law index, but with different intensities. Due to the lack of statistics of listed events with the same power-law index, we "regenerate" a set of events by fixing the intensity of each SEP spectra at $500 \mathrm{MeV}$, while forcing all spectra to have the same power-law index, e.g., $\gamma=-2$. The choice of the anchor point $500 \mathrm{MeV}$ is arbitrary, as this is just helping to regenerate the spectra with similar intensities; it could also be 200 or $300 \mathrm{MeV}$. The regenerated spectral indices and the particle energy spectra are shown in Figure 1.

Apart from the SEP intensity and spectral index, we also investigate the influence of the energy ranges on the surface radiation. In Guo et al. (2018b), for a few historical events, we calculated the surface dose rate resulting from 100 to $800 \mathrm{MeV}$ primary protons is about $93 \%$ of that induced by the full SEP spectra. This is because of the effective atmospheric shielding of particles with low energies ${ }^{8}$ and the nature of the SEP spectra where the high-energy component has significantly less flux. In this study, we first calculate the Martian surface radiation induced by SEP spectra from 100 to $800 \mathrm{MeV}$ as used for fitting the SEP events by Kühl et al. (2017). We then test the influence of the SEP energy range on the surface radiation using three different cases: $15-1000 \mathrm{MeV}, 15-1500 \mathrm{MeV}$, and $15-2000 \mathrm{MeV}$. Based on these spectra with different properties, we model the induced Martian surface radiation, dose rate, and dose-equivalent rate by each event as shown in Section 3 .

\footnotetext{
8 As shown in Figure 2 in Guo et al. (2019b), there is a sharp decrease of surface dose contribution from primary protons with energies below $\sim 165$ $\mathrm{MeV} \pm 30 \mathrm{MeV}$ (depending on the elevation of interest and the varying seasonal pressure), which can be considered to be the atmospheric cutoff energy.
} 

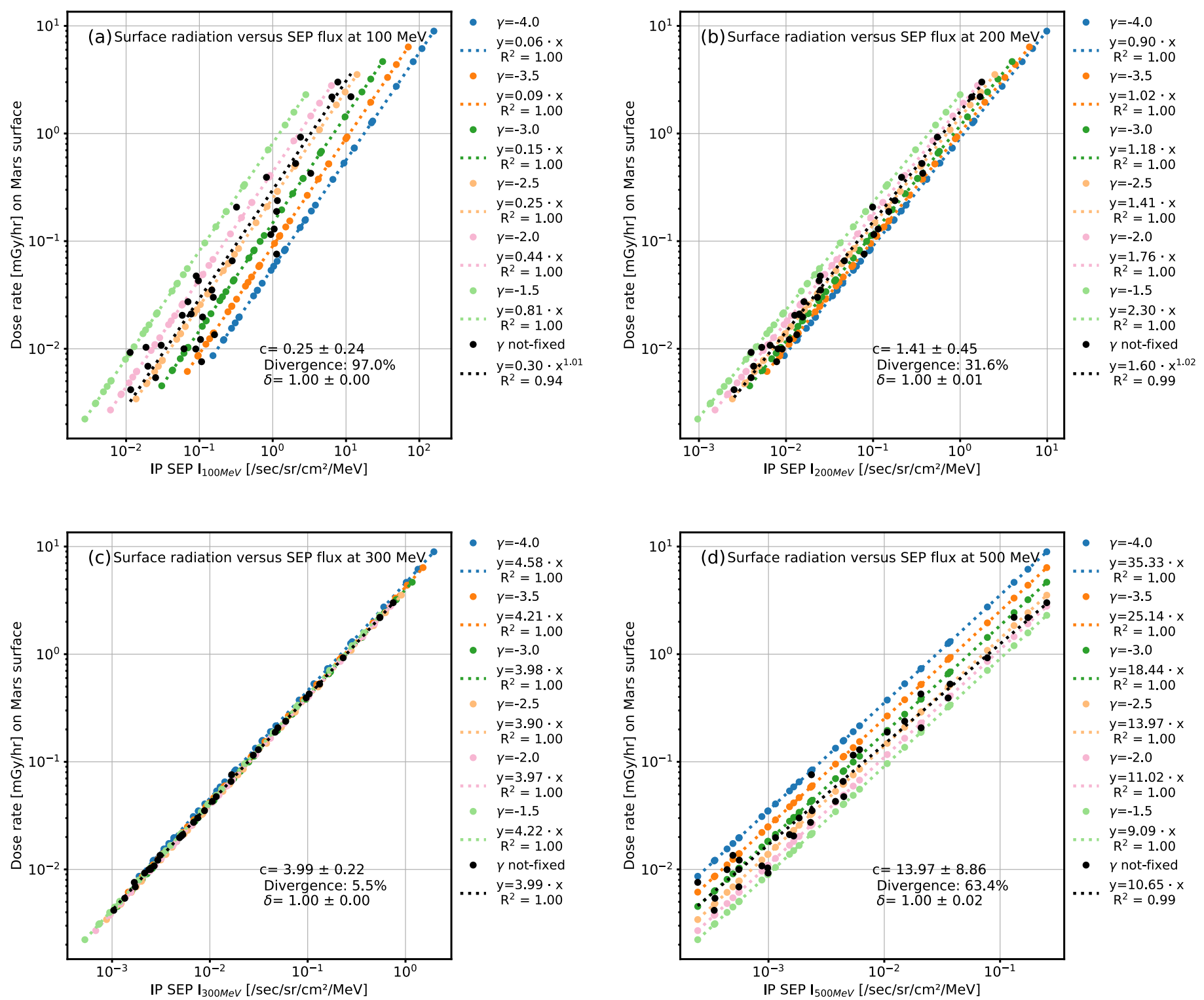

Figure 2. Martian surface dose rate resulting from primary SEP spectra (100-800 MeV protons) vs. primary SEP flux at different energies on top of the Martian atmosphere. Black dots are the calculations based on actual events from the list of Kühl et al. (2017), and each different color represents a fixed power-law index $\gamma$. Data points of the same color in each panel are fitted with $y=c x^{\delta}$ with the fitted parameters and their deviations shown in the legends.

\section{Results and Discussions}

Figure 2 shows the SEP-induced Martian surface dose rate (from primary $100-800 \mathrm{MeV}$ protons) versus the primary SEP flux at certain energies (e.g., 100, 200, 300, and $500 \mathrm{MeV}$ in each panel). As shown, when the power-law spectral index is the same for different events, e.g., $\gamma=-4$, the induced Martian surface dose rate and the SEP flux at certain energy are marked as blue dots and fitted with blue dashed lines in each panel of the figure. Due to the large span of the $x$ and $y$ values in Figure 2, we plot and fit the results in logarithmic scale $y=c x^{\delta}$. However as indicated by the fitted index $\delta \sim 1$, the correlation is very close to a perfect linear function. For different power-law $\gamma$ indices, the linear correlations between the surface dose rate and the SEP flux at a certain energy are generally different. For instance, at $100 \mathrm{MeV}$, as shown in panel (a), the fitted linear coefficient $c$ is different from one another and the divergence (ratio of the standard deviation and the mean value) is as large as $97 \%$. This is expected: a power- law SEP spectrum depends on two parameters and when the $\gamma$ value is fixed, the induced dose rate should be proportional to the SEP intensity (i.e., $\delta=1$ ); when spectral $\gamma$ values are different, the dependence of the dose rate on the intensity, $c$, also changes. Surprisingly, at $300 \mathrm{MeV}$, the divergence dramatically decreases to only $5.5 \%$ (shown as a converging line of all different colors in panel (c)), meaning that the correlation between the surface dose rate and the SEP flux is almost independent on the SEP spectral index $\gamma$. In other words, for an SEP spectrum which can be fitted with a powerlaw distribution between 100 and $800 \mathrm{MeV}$, its intensity at $300 \mathrm{MeV}$ alone can determine the Martian surface radiation dose rate.

To test the robustness of this result, we also carried out the above study using other ranges of the incident SEP spectra, i.e., $15-1000 \mathrm{MeV}$, $15-1500 \mathrm{MeV}$, and also $15-2000 \mathrm{MeV}$ to account for the remaining contribution to the surface radiation by particles below $100 \mathrm{MeV}$ (which can still slightly induce the surface dose via generating some secondary particles arriving at 

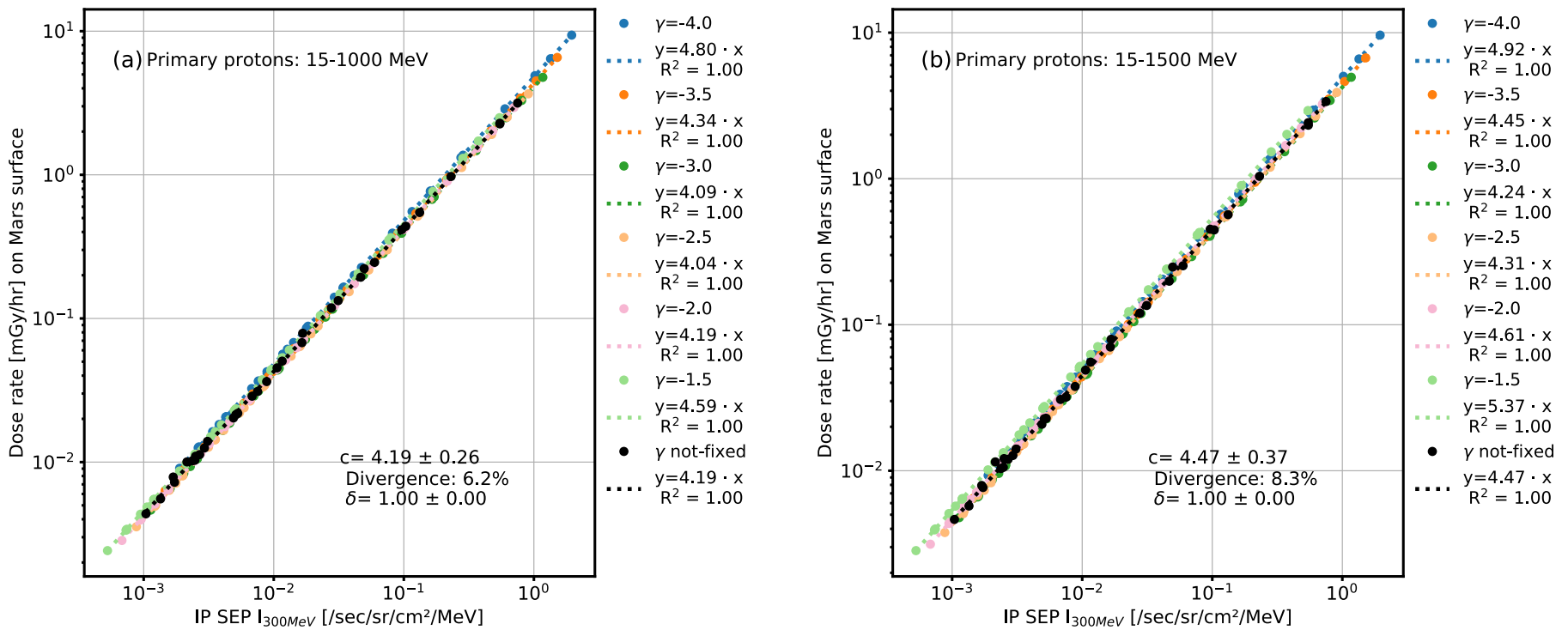

Figure 3. Martian surface dose rate vs. primary SEP flux at $300 \mathrm{MeV}$ for primary power-law spectra ranging from (a) $15-1000 \mathrm{MeV}$ protons and (b) $15-1500 \mathrm{MeV}$ protons. Color features of the figure are the same as in Figure 2.

the surface, especially neutrons and gammas; Guo et al. 2019b) or above $800 \mathrm{MeV}$ when such particles are present during extreme events, especially with flat power-law spectra (small $|\gamma|$ values). The correlation of the $300 \mathrm{MeV}$ SEP intensity and the Martian surface dose rate is still very good for the three test cases (and Figure 3 shows the results of the first two cases), and the fitted correlation coefficients are rather consistent with that of the $100-800 \mathrm{MeV}$ range shown in Figure 2. Considering all four cases of energy ranges, we formulate the correlation between SEP intensity [particles $\mathrm{s}^{-1} \mathrm{sr}^{-1} \mathrm{~cm}^{-2} \mathrm{MeV}^{-1}$ ] at $300 \mathrm{MeV}$ and the Martian surface radiation dose rate $[\mathrm{mGy} /$ hour] as follows:

$$
D_{\text {Mars }}=4.45 \cdot I_{300 \mathrm{MeV}}( \pm 9.8 \%),
$$

where the coefficient 4.45 has the unit of $(\mathrm{mGy} / \mathrm{hr})$. $\left(\mathrm{s} \mathrm{sr} \mathrm{cm}{ }^{2} \mathrm{MeV}\right)$ and is the mean value of the fitted parameters from all cases calculated (four different energy ranges, each with seven different power-law indices). The $9.8 \%$ error bar accounts for the divergence of fitted parameters from different cases. We note that the distribution of all coefficients is slightly different from Gaussian, and the median value is 4.33 , smaller than the mean value. The upper and lower boundaries of the coefficients from all cases are 5.87 and 3.90, respectively.

In terms of biological effectiveness associated with radiation exposures on human beings, the dose equivalent is often more referred to for evaluating the deep-space exploration risks (Mountford \& Temperton 1992). Therefore, we have also explored the correlation between SEP properties, varying among different energy ranges, intensities, and power-law indices and the dose equivalent, and we find the $300 \mathrm{MeV}$ SEP flux [particles $\mathrm{s}^{-1} \mathrm{sr}^{-1} \mathrm{~cm}^{-2} \mathrm{MeV}^{-1}$ ] still being the pivot point for the surface dose-equivalent rate $[\mathrm{mSv} /$ hour] following this correlation:

$$
E_{\mathrm{Mars}}=5.56 \cdot I_{300 \mathrm{MeV}}( \pm 12.9 \%),
$$

where the coefficient 5.56 is the mean value of all cases studied and has the unit of $(\mathrm{mSv} / \mathrm{hr}) \cdot\left(\mathrm{s} \mathrm{sr}^{2} \mathrm{MeV}\right)$.

The error bars in Equations (1) and (2) are no bigger than the uncertainties, which are between 5\% and 15\%, resulting from the choice of different atmospheric properties, particle transport models through the Martian atmosphere, and physics lists included therein as discussed in Matthiä et al. (2016) and Guo et al. (2019a). Due to this strong linear correlation between the SEP flux at $300 \mathrm{MeV}$ and the Martian surface dose rate and dose-equivalent rate, we name the $300 \mathrm{MeV}$ as the pivot energy of SEPs contributing to the Martian surface radiation. This can be explained by the balance between the contribution to the surface dose from particles below $300 \mathrm{MeV}$ and those above. For instance, when changing the spectral index $\gamma$ from -4 to -2 , the reduction of the dose contribution from particles below $300 \mathrm{MeV}$ is compensated by the increased dose from particles above this pivot point. Alternatively, let us consider the primary proton contribution to the Martian surface dose as a function of the incoming particle energy that has been calculated and shown in Figure 2 of Guo et al. (2019b). The function shows that primary protons below $\sim 160 \mathrm{MeV}$ have little effect on the Martian surface radiation. For an SEP event, we need to fold its spectra with this energy-dependent function for calculating the surface dose rate. The combination of an SEP spectrum, which often has a power-law distribution at energies above $\sim 10-30 \mathrm{MeV}$, with the Martian atmospheric function leading to an energy point at which the dose contributions below and above this point are balanced out no matter how the spectral index changes. In Appendix B we show the analytical derivation of the pivot energy of the dose rate induced by power-law SEP spectra.

We note that this result is limited to power-law-shaped large SEPs with proton energy extending to above $\sim 500 \mathrm{MeV}$ as a common feature of the sampled events for studying the correlation (Kühl et al. 2017). This empirical correlation will likely not work for SEP events with only protons of energies smaller than $\sim 500 \mathrm{MeV}$ or for events with distributions far away from a power-law shape at these high energies. We have investigated the influence of non-power-law spectra on the correlation using a few historical events: the 1989 October and September and 1972 August events, for which the proton distributions have been constructed using a Weibull function (Townsend et al. 2006). For each of these three events, we calculate the Martian surface dose rate induced by the whole 
input spectrum and compare it with that estimated by our pivotenergy correlation shown in Equation (1). For the two 1989 events, although the SEP spectra do not have a single power law through all energies, particles distributed between $\lesssim 100 \mathrm{MeV}$ and $1 \mathrm{GeV}$ can be reasonably fitted by a power law with $\gamma$ indices around -2.2 (Guo et al. 2018b). The surface dose rates estimated using the pivot-energy correlation are about $9 \%$ and $6 \%$ larger than those calculated from the complete spectra for the 1989 October and September events, respectively. The overestimation is because the actual spectra, different from a single power law, fall off slightly at both low$(\lesssim 30 \mathrm{MeV})$ and high-energy $(\gtrsim 1 \mathrm{GeV})$ ends. However the pivot-energy estimation is still consistent with the actual surface radiation within uncertainties. Alternatively, the 1972 August spectral shape is rather unusual with a large initial flux at energies below $\sim 100 \mathrm{MeV}$ (almost a flat power law with $\gamma$ between 0 and -1 ) followed by a very sharp fall-off at higher energies; particle flux between 100 and $800 \mathrm{MeV}$ cannot be described by any power-law distribution while those above $500 \mathrm{MeV}$ up to $1 \mathrm{GeV}$ can be fitted by an exceptionally soft power law with an index of -38 . The surface radiation estimated using the $300 \mathrm{MeV}$ pivot-energy correlation is about 17 times smaller than that resulting from the complete spectrum, which is explainable by the characteristics of the spectra where the majority of the event flux is contributed by particles below $\sim 200 \mathrm{MeV}$. This highlights the importance and necessity of continuing Martian surface radiation measurement by MSL/RAD for nowcasting any potentially hazardous radiation enhancement due to the unpredictable nature of SEPs.

Another additional caveat is that the Martian atmospheric depth used in the current model is equivalent to the average value of $\sim 22 \mathrm{~g} \mathrm{~cm}^{-2}$ measured at Gale Crater by the Curiosity rover. This depth was chosen for the purpose of model validation (more in Appendix A) using MSL/RAD. The atmospheric shielding may be slightly smaller at other highaltitude places for which the correlation obtained here needs to be reevaluated. Thus, further benchmarks of the model using in situ RAD measurements at different atmospheric depth, especially as Curiosity is climbing up to Mount Sharp, would be needed. Long-term measurements of the radiation environment on Mars under different solar modulation and atmospheric conditions would also favor the validation of various radiation transport models that may differ by up to an order of magnitude (Matthiä et al. 2017).

Besides, the dose and dose equivalent given here are based on a "biological phantom" representing some thin-skin structures and cannot be directly applied to phantoms such as detectors with different materials or body organs embedded under different body depths. Nevertheless, such a simplified and elegant quantification can serve to make instant predictions, within $1 \mathrm{~ms}$, of the radiation environment on Mars upon the onset of large SEP events where protons are accelerated up to more than $500 \mathrm{MeV}$.

For the purpose of mitigating radiation risks for future Mars missions, a particle detector sensitive to protons at these specific energies (i.e., at $300 \mathrm{MeV}$ and perhaps also a channel for $E>500 \mathrm{MeV}$ to detect if particles reached the high-energy range) can be located in the inner heliosphere with a good magnetic connection to Mars in order to provide an alert ( $\sim$ hours ahead) for potential hazardous radiation environment on Mars. However, the $300 \mathrm{MeV}$ particle flux at Mars (needed as our model input) will be likely smaller than that at Earth orbit due to the propagation and scattering of particles as they are transported outward in the heliosphere. Thus, we need detailed knowledge of the acceleration and injection profiles of the particles (e.g., Lario et al. 2017) as well as the heliospheric structure in the interplanetary space that may influence the particle propagation toward Mars (e.g., Guo et al. 2018a) to achieve more precise forecasting. Such knowledge, especially for the Mars direction, is often not available and thus difficult to be properly included in a timely forecast model. However, in light of much more detailed information on solar eruptions and particle propagations provided by Parker Solar Probe (Fox et al. 2016) and Solar Orbiter (Mueller et al. 2013) in the near future, more reliable data-driven SEP injection and interplanetary transport models could be used to make better predictions of the SEP flux at Mars, allowing us to provide instantaneous and quantitative alerts for future human missions at Mars upon the onset of large SEP events at the Sun.

R.A.D. is supported by NASA (HEOMD) under Jet Propulsion Laboratory (JPL) subcontract 1273039 to Southwest Research Institute and in Germany by the German Aerospace Center (DLR) and DLRs Space Administration grants 50QM0501, 50QM1201, and 50QM1701 to the Christian-Albrechts-University, Kiel. J.G. and Y.W. are supported by the Key Research Program of the Chinese Academy of Sciences (grant Nos. XDPB11 and QYZDB-SSWDQC015) and the NSFC (grant No. 41842037). J.G., R.F.W.S., and M.G. acknowledge the International Space Science Institute, which made part of the collaborations in this Letter through the ISSI International Team 353 "Radiation Interactions at Planetary Bodies."

\section{Appendix A Model Implementation for Calculating the Martian Surface Radiation Environment}

For calculating the particle flux and radiation level on the surface of Mars, we need to consider the physical processes by which primary particles arriving at Mars interact with the Martian atmosphere. Generally speaking, primary particles (GCRs or SEPs) that reach Mars may undergo elastic or inelastic nuclear interactions with atmospheric nuclei, losing energy and, in inelastic reactions, creating secondary particles via spallation and fragmentation processes. These secondary particles may further interact with the atmosphere as they propagate downward and even with the Martian regolith, and finally result in complex spectra including both primaries and secondaries at the surface of Mars. There are various particle transport codes such as HZETRN (Slaba et al. 2016; Wilson et al. 2016), PHITS (Sato et al. 2013), and GEANT4/ PLANETOCOSMICS (Desorgher et al. 2006) and the newly developed GEANT4/AtRIS tool (Banjac et al. 2019), which can be employed for calculating the interaction of particles with the Martian atmospheric and regolith environment.

These transport models need to be implemented with a model containing Martian atmosphere and regolith properties, shown as the second row in the third column of the flowchart shown in Figure 4. In particular, the Mars Climate Database (MCD ${ }^{9}$; Lewis et al. 1999) offers location- and time-dependent descriptions of the Martian atmospheric properties, such as temperature, density, composition, etc. In the current model

\footnotetext{
http://www-Mars.lmd.jussieu.fr
} 


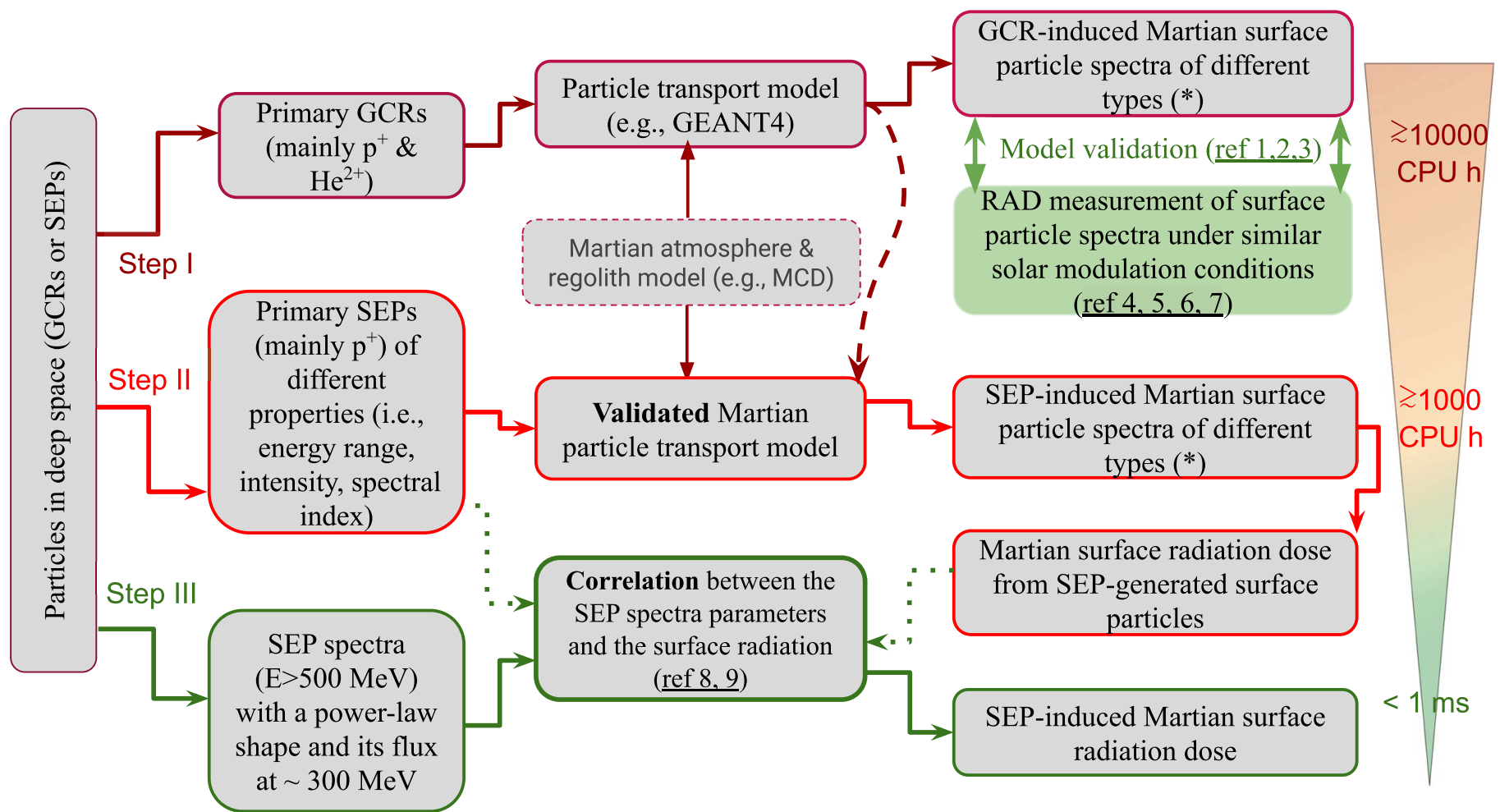

Figure 4. Flowchart of the implementation of the models for calculating the Martian surface radiation environment as used in the current and relevant studies. Step I includes the model setup and validation. Step II applies the above validated model to alarmed SEP events with different properties. Step III employs the parameterized correlation between SEP spectral property and the surface radiation environment based on Step II analysis to quickly derive the Martian surface radiation based on minimized SEP spectral input. ( $\left.{ }^{*}\right)$ Surface secondary particles considered in the model output include proton, electron, position, ${ }^{4} \mathrm{He},{ }^{3} \mathrm{He}$, deuteron, triton, neutron, and gamma above $1 \mathrm{MeV}$. References 1, 2, 3 for model validations are Matthiä et al. (2016, 2017), Guo et al. (2019a). References 4, 5, 6, 7 for RAD surface spectra measurements are Ehresmann et al. (2014, 2017), Köhler et al. (2014), Guo et al. (2017b). References 8 and 9 for extracted correlations are Guo et al. (2018b) and this study. The approximate computing central processing unit (CPU) time is marked in the rightmost part. More explanations and descriptions of this chart can be found in the text.

setup, we use the atmosphere profiles from MCD above the ground at the location of Gale Crater, which is the landing site of the Curiosity rover, in order to validate the modeled surface radiation field with actual RAD measurement, as discussed later. More detailed descriptions of features of the MCD implemented in the model used here can be found in Guo et al. (2018b, 2019a) and Appel et al. (2018).

Based on particle transport models implemented with the Martian environment features, the radiation on Mars can be calculated. Previous works have studied the Martian surface radiation under different conditions, e.g., at different geographic locations (e.g., Saganti et al. 2004), under various Martian atmospheric environments such as during dust storms (e.g., Norman et al. 2014) or at different atmospheric depth (Guo et al. 2017a) or in the Martian soil (Dartnell et al. 2007), with GCRs under different solar modulation conditions (e.g., Simonsen \& Nealy 1993), with SEPs of different spectra (e.g., Townsend et al. 2006; Guo et al. 2018b), etc. Comparison of GEANT4 and HZETRN for calculating the GCR radiation environment on Mars has shown consistent results from both models (Gronoff et al. 2015). However, only recently have the surface particle spectra and dose detected by RAD allowed for direct validation of such models with actual measurements (second row of the last column in Figure 4). Particularly relevant for the current study, the GEANT4 model has been shown to predict the surface particle spectra with good accuracy for many (such as hydrogen and helium isotopes and heavy ions), but not all aspects (such as neutrons), as validated by RAD measurements of the GCR-induced radiation (Matthiä et al. 2016, 2017; Guo et al. 2019a). The abovedescribed model setup, implementation, and validation are summarized as Step I in Figure 4. To meet the requirement of the statistics of the modeling results, especially from ions heavier than protons, the computational time needed could be as large as 10,00o CPU h or more.

Based on the validated GEANT4 particle transport model through the Martian atmosphere, we have calculated the induced Martian surface radiation environment of more than 30 significant solar events (measured in situ at Earth), in order to provide insights into the possible variety of Martian surface radiation environments that may be induced during large SEP events (Guo et al. 2018b). Depending on the intensity and shape of the solar particle spectra incident at the top of the atmosphere, as well as the distribution of particle types, different SEP events lead to rather different radiation environments on the surface of Mars. We first studied some historical SEP events and their induced radiation on Mars, i.e., deposited dose rate and dose-equivalent rate in a $0.5 \mathrm{~mm}$ water slab accumulated from surface secondary particle spectra of various types (proton, electron, position, ${ }^{4} \mathrm{He},{ }^{3} \mathrm{He}$, deuteron, triton, neutron, gamma, etc.) induced by an SEP event. According to the International Commission on Radiological Protection recommendations (Mountford \& Temperton 1992), the dose equivalent is the reweighted dose by a quality factor $Q$ that is a function of Linear Energy Transfer (energy deposited per distance). We found that the surface dose or dose-equivalent 
rates do not depend significantly on the full primary spectra, which could range from a few $\mathrm{keV}$ up to several hundred $\mathrm{MeV}$ (occasionally even reaching 1 or $2 \mathrm{GeV}$ or further above). For both the 1989 October and September events that have energetic protons up to about $2 \mathrm{GeV}$, the surface dose rate resulting from 15 to $1000 \mathrm{MeV}$ primary protons is about $96 \%$ of that from the full SEP spectra; the surface dose rate resulting from 100 to $800 \mathrm{MeV}$ primary protons is about $93 \%$ of that induced by the full SEP spectra. This is because of the effective atmospheric shielding of particles with low energies (protons below about $150 \mathrm{MeV}$ hardly penetrate down to the surface depth at $\sim 20 \mathrm{~g} \mathrm{~cm}^{-2}$ ) and the nature of the SEP spectra where the high-energy component has significantly less flux. The above implementation of the validated model to calculate the SEP-induced Martian surface radiation is summarized as Step II in Figure 4.

In this study, we calculate the induced Martian surface radiation by a variety of SEP events with different properties such as their energy range, intensity, power-law index, etc., as described in the main text. Then we analyze and correlate the parameterized properties of the SEP spectra and the resulting surface dose rate of each event in order to quantify the Martian surface radiation based on SEP spectral properties. We find that at the pivot energy of $\sim 300 \mathrm{MeV}$, the SEP intensity alone can determine the surface dose rate. This correlation minimizes the computational power of deriving the SEP-induced Martian surface radiation level to less than $1 \mathrm{~ms}$, shown as Step III in Figure 4. The result and discussion of the pivot-energy study are elaborated in the main text.

\section{Appendix B \\ Mathematical Derivation of the Pivot Energy}

Any given SEP power-law spectrum can be written as

$$
I(E)=I_{0} E^{\gamma} .
$$

For certain energy, the above function can be rewritten as

$$
I(E)=I_{E_{i}}\left(\frac{E}{E_{i}}\right)^{\gamma}
$$

where $I_{E i}$ is the SEP intensity at certain energy $E_{i}$, e.g., the $x$ axes of SEP intensity at $100,200,300$, or $500 \mathrm{MeV}$ as shown in Figure 2(a)-(d). For a given power-law index $\gamma$, the calculated Martian surface dose rate can be fitted linearly proportional to the SEP intensity at a certain energy with a function $y=c \cdot x$, as shown in Figure 2. For the same event (fixed gamma), we obtain a certain surface dose rate so that

$$
c_{i} I_{E_{i}}=c_{j} I_{E_{j}}
$$

Combining the above two equations, we obtain

$$
\frac{c_{i}}{c_{j}}=\left(\frac{E_{i}}{E_{j}}\right)^{-\gamma}
$$

Applying the above equation with two different power-law indices $\gamma_{1}$ and $\gamma_{2}$, we have

$$
\begin{aligned}
& c_{i 1}=c_{j 1}\left(\frac{E_{i}}{E_{j}}\right)^{-\gamma_{1}} \\
& c_{i 2}=c_{j 2}\left(\frac{E_{i}}{E_{j}}\right)^{-\gamma_{2}} .
\end{aligned}
$$

For the existence of a pivot energy at $E_{i}$, we expect the correlation coefficient to be constant for different $\gamma$ values, i.e., $c_{i 1}=c_{i 2}$. Implementing this relation to Equation (7), we obtain

$$
E_{p} \equiv E_{i}=\left(\frac{c_{j 1}}{c_{j 2}}\right)^{-\left(\gamma_{1}-\gamma_{2}\right)} E_{j} .
$$

This means that for a given SEP energy, e.g., $E_{j}$, with two coefficients $c_{j 1}$ and $c_{j 2}$ derived under two different power-law indices we can obtain the pivot energy $E_{i}$, also defined as $E_{p}$. For instance, using values of $\gamma_{1}=-2$ and $\gamma_{2}=-3$ as well as $c_{j 1}=11.02$ and $c_{j 2}=18.44$ for the SEP intensity at $E_{j}=500$, as shown in Figure 2(d), we can derive $E_{i}$ to be $299 \mathrm{MeV}$, $\sim 300 \mathrm{MeV}$. Such estimations based on different $E_{j}$ and fitted $c_{j}$ may differ from one another. But the average $E_{p}$ is around $300 \mathrm{MeV}$.

\section{ORCID iDs}

Jingnan Guo (i) https://orcid.org/0000-0002-8707-076X Robert F. Wimmer-Schweingruber (1) https://orcid.org/00000002-7388-173X

Yuming Wang (ํ) https://orcid.org/0000-0002-8887-3919

Manuel Grande (ib https://orcid.org/0000-0002-2233-2618

Daniel Matthiä (i) https://orcid.org/0000-0003-1507-0143

Cary Zeitlin (1) https://orcid.org/0000-0002-1737-141X

Bent Ehresmann (1) https://orcid.org/0000-0002-5956-5722

Donald M. Hassler (1) https://orcid.org/0000-0001-8830-1200

\section{References}

Appel, J. K., Köehler, J., Guo, J., et al. 2018, E\&SS, 5, 2

Banjac, S., Herbst, K., \& Heber, B. 2019, JGRA, 124, 50

Cucinotta, F. A., \& Durante, M. 2006, The Lancet Oncology, 7, 431

Dartnell, L., Desorgher, L., Ward, J., \& Coates, A. 2007, GeoRL, 34, L02207

Desorgher, L., Flückiger, E. O., \& Gurtner, M. 2006, 36th COSPAR Scientific Assembly, CDROM abstract \#2361

Ehresmann, B., Hassler, D., Zeitlin, C., et al. 2018, GeoRL, 45, 5305

Ehresmann, B., Zeitlin, C., Hassler, D. M., et al. 2014, JGRE, 119, 468

Ehresmann, B., Zeitlin, C. J., Hassler, D. M., et al. 2017, LSSR, 14, 3

Fox, N. J., Velli, M. C., Bale, S. D., et al. 2016, SSRv, 204, 7

Gronoff, G., Norman, R. B., \& Mertens, C. J. 2015, AdSpR, 55, 1799

Grotzinger, J. P., Crisp, J., Vasavada, A. R., et al. 2012, SSRv, 170, 5

Guo, J., Dumbović, M., Wimmer-Schweingruber, R. F., et al. 2018a, SpWea, 16,1156

Guo, J., Sanjac, S., Röstel, L., et al. 2019a, JSWSC, 9, A2

Guo, J., Slaba, T. C., Zeitlin, C., Wimmer-Schweingruber, R. F., et al. 2017a, JGRE, 122, 329

Guo, J., Wimmer-Schweingruber, R. F., Grande, M., Lee-Payne, Z. H., \& Matthiä, D. 2019b, JSWSC, 9, A7

Guo, J., Zeitlin, C., Wimmer-Schweingruber, R., et al. 2017b, LSSR, 14, 12

Guo, J., Zeitlin, C., Wimmer-Schweingruber, R. F., et al. 2018b, AJ, 155, 49

Hassler, D., Zeitlin, C., Ehresmann, B., et al. 2018, SpWea, 16, 1702

Hassler, D., Zeitlin, C., Wimmer-Schweingruber, R., et al. 2012, SSRv, 170,503

Hassler, D. M., Zeitlin, C., Wimmer-Schweingruber, R. F., et al. 2014, Sci, 343, 1244797

Köhler, J., Zeitlin, C., Ehresmann, B., et al. 2014, JGRE, 119, 594 
Kühl, P., Dresing, N., Heber, B., \& Klassen, A. 2017, SoPh, 292, 10 Lario, D. 2005, AdSpR, 36, 2279

Lario, D., Kwon, R.-Y., Richardson, I. G., et al. 2017, ApJ, 838, 51 Lewis, S. R., Collins, M., Read, P. L., et al. 1999, JGRE, 104, 24177 Matthiä, D., Ehresmann, B., Lohf, H., et al. 2016, JSWSC, 6, A13 Matthiä, D., Hassler, D. M., de Wet, W., et al. 2017, LSSR, 14, 18

Mountford, P. J., \& Temperton, D. H. 1992, European Journal of Nuclear Medicine, 19, 77

Mueller, D., Marsden, R. G., Cyr, O. S., et al. 2013, SoPh, 285, 25

Norman, R. B., Gronoff, G., \& Mertens, C. J. 2014, JGRA, 119, 452

Parker, E. 1958, PhFl, 1, 171
Saganti, P. B., Cucinotta, F. A., Wilson, J. W., Simonsen, L. C., \& Zeitlin, C. 2004, SSRv, 110, 143

Sato, T., Niita, K., Matsuda, N., et al. 2013, Journal of Nuclear Science and Technology, 50, 913

Simonsen, L. C., \& Nealy, J. E. 1993, NASA Technical Paper Series, 3300

Simpson, J. 1983, ARNPS, 33, 323

Slaba, T. C., Wilson, J. W., Badavi, F. F., Reddell, B. D., \& Bahadori, A. A. 2016, LSSR, 9, 77

Townsend, L. W., Stephens, D. L., Hoff, J., et al. 2006, AdSpR, 38, 226

Wilson, J. W., Slaba, T. C., Badavi, F. F., Reddell, B. D., \& Bahadori, A. A 2016, LSSR, 9, 69

Zeitlin, C., Hassler, D., Guo, J., et al. 2018, GeoRL, 45, 5845 